\title{
1 SQuIRE: Software for Quantifying Interspersed Repeat Elements
}

2 Authors: Yang Wan R. ${ }^{1}$, Daniel Ardeljan ${ }^{1,2}$, Clarissa N. Pacyna ${ }^{1,3}$, Lindsay M. Payer ${ }^{1,6}$, Kathleen H.

3 Burns $s^{1,3,4,5,6}$

$4{ }^{1}$ Department of Pathology, Johns Hopkins University School of Medicine, Baltimore, Maryland, 21205,

5 USA.

$6{ }^{2}$ McKusick-Nathans Institute of Genetics, Johns Hopkins University School of Medicine, Baltimore,

7 Maryland, 21205, USA.

$8{ }^{3}$ Thomas C. Jenkins Department of Biophysics, Johns Hopkins University, Baltimore, Maryland, USA

$9{ }^{4}$ Department of Oncology, Johns Hopkins University School of Medicine, Baltimore, Maryland, USA.

$10{ }^{5}$ Sidney Kimmel Comprehensive Cancer Center, Johns Hopkins University School of Medicine,

11 Baltimore, Maryland, USA.

$12{ }^{6}$ These authors contributed equally to this work.

13 Running Title: SQuIRE

14 Keywords: TE, retrotransposon, mobile element, RNA-seq alignment, differential expression

15 analysis

16 Corresponding author:Kathleen H. Burns, M.D., Ph.D.

17 Johns Hopkins University School of Medicine

18733 N. Broadway, MRB 447

19 Baltimore, MD 21205

20 kburns@jhmi.edu

$21 \quad 410-502-7214$ 


\section{Abstract:}

Transposable elements are interspersed repeat sequences that make up much of the human

24 genome. Conventional approaches to RNA-seq analysis often exclude these sequences, fail to

25 optimally adjudicate read alignments, or align reads to interspersed repeat consensus sequences

26 without considering these transcripts in their genomic contexts. As a result, repetitive sequence

27 contributions to transcriptomes are not well understood. Here, we present Software for

28 Quantifying Interspersed Repeat Expression (SQuIRE), an RNA-seq analysis pipeline that

29 integrates repeat and genome annotation (RepeatMasker), read alignment (STAR), gene

30 expression (StringTie) and differential expression (DESeq2). SQuIRE uniquely provides a locus-

31 specific picture of interspersed repeat-encoded RNA expression. SQuIRE can be downloaded at

32 (github.com/wyang17/SQuIRE).

\section{Introduction}

Transposable elements (TEs) are self-propagating mobile genetic elements. Their insertions have resulted in a complex distribution of interspersed repeats comprising almost half of the human genome (Lander et al. 2001; Kazazian 2004). They propagated through either DNA ('transposons') or RNA

37 intermediates ('retrotransposons')(Huang et al. 2012; Burns and Boeke 2012). Retrotransposons are

38 further classified into Orders based on the presence of long terminal repeats (LTR retrotransposons) or

39 whether they were long or short interspersed elements (LINEs and SINEs)(Wicker et al. 2007). Although

40 most TEs have lost the capacity for generating new insertions over their evolutionary history and are now

41 fixed in the human population, a subset of younger subfamilies from the LINE-1 superfamily (i.e., L1PA1

42 or L1HS) (Beck et al. 2011), the SINE Alu superfamily (e.g., AluYa5, AluYa8, AluYb8, AluYb9)

43 (Deininger 2011), and composite SVA (SINE-variable number tandem repeat (VNTR)-Alu) elements

44 (Hancks et al. 2010) remain retrotranspositionally active and generate new polymorphic insertions

45 (Stewart et al. 2011; Abecasis et al. 2012). 
Due to the repetitive nature of TEs, short-read RNA sequences that originate from one locus can

47 ambiguously align to multiple copies of the same subfamily dispersed throughout the genome. This

48 problem is most significant for younger TEs; older elements have accumulated nucleotide substitutions

49 over millions of years that can differentiate them and give rise to uniquely aligning TE reads (Giordano et

50 al. 2007). Because of these barriers, conventional RNA-seq analyses of TEs have either discarded multi-

51 mapping alignments (Chuong et al. 2013) or combined TE expression to the subfamily level (Criscione et

52 al. 2014; Jin et al. 2015; Lerat et al. 2016). Other groups have studied active LINE-1s using tailored

53 pipelines, leveraging internal sequence variation and 3' transcription extensions into unique sequence

54 (Philippe et al. 2016; Deininger et al. 2017; Scott et al. 2016). However, these targeted approaches are

55 unable to provide a comprehensive picture of TE expression.

To analyze global TE expression in conventional RNA-seq experiments, we have developed

57 the Software for Quantifying Interspersed Repeat Elements (SQuIRE). SQuIRE is the first RNA-seq

58 analysis pipeline available to date that quantifies TE expression at the locus level. In addition to RNA-seq

59 providing expression estimations at the TE locus level, SQuIRE quantifies expression at the subfamily

60 level and performs differential expression analyses on TEs and genes. We benchmark our pipeline using

61 both simulated and experimental datasets and compare its performance against other software pipelines

62 designed to quantify TE expression (Criscione et al. 2014; Jin et al. 2015; Lerat et al. 2016). SQuIRE

63 provides a suite of tools to ensure the pipeline is user-friendly, reproducible, and broadly applicable.

$64 \quad$ Results

\section{SQuIRE Overview}

SQuIRE provides a suite of tools for analyzing transposable element (TE) expression in RNA-seq

67 data (Fig. 1). SQuIRE's tools can be organized into four stages: 1) Preparation, 2) Quantification, 3)

68 Analysis and 4) Follow-up. In the Preparation stage, Fetch downloads requisite annotation files for any

69 species with assembled genomes available on University of California Santa Cruz (UCSC) Genome 
70 Browser (Kent et al. 2002). These annotation files include RefSeq (Pruitt et al. 2014) gene information in

71 BED and GTF format, and RepeatMasker (Smit, AFA, Hubley, R \& Green) TE information in a custom

72 format. Fetch also creates an index for the aligner STAR (Dobin et al. 2013) from chromosome FASTA

73 files. Clean reformats TE annotation information from RepeatMasker into a BED file for downstream

74 analyses. The tools in the Preparation stage only need to be run once per genome build. Because there are

75 multiple RNA-seq aligners that can produce different results for TE expression estimation, the

76 Quantification stage includes the alignment step Map to ensure reproducibility. Map aligns RNA-seq

77 data using the STAR aligner with parameters tailored to TEs that allow for multi-mapping reads and

78 discordant alignments. It produces a BAM file. Count quantifies TE expression using a SQuIRE-specific

79 algorithm that incorporates both unique and multi-mapping reads. It outputs read counts and fragments

80 per kilobase transcript per million reads (fpkm) for each TE locus, and aggregates TE counts and fpkm for

81 TE subfamilies into a separate file. Count also quantifies annotated RefSeq gene expression with the

82 transcript assembler StringTie (Pertea et al. 2015) to output annotated gene expression as fpkm in a GTF

83 file, and as counts in a count table file. In the Analysis stage, Call performs differential expression

84 analysis for TEs and RefSeq genes with the Bioconductor package DESeq2 (Love et al. 2014; Huber et al.

85 2015). To allow users to visualize alignments to TEs of interest visualized by the Integrative Genomics

86 Viewer (IGV)(Robinson et al. 2011) or UCSC Genome Browser, the Follow-up stage tool Draw creates

87 bedgraphs for each sample. Seek retrieves sequences for genomic coordinates supplied by the user in

88 FASTA format. 
bioRxiv preprint doi: https://doi.org/10.1101/313999; this version posted May 4, 2018. The copyright holder for this preprint (which was not certified by peer review) is the author/funder, who has granted bioRxiv a license to display the preprint in perpetuity. It is made available under aCC-BY-NC 4.0 International license.

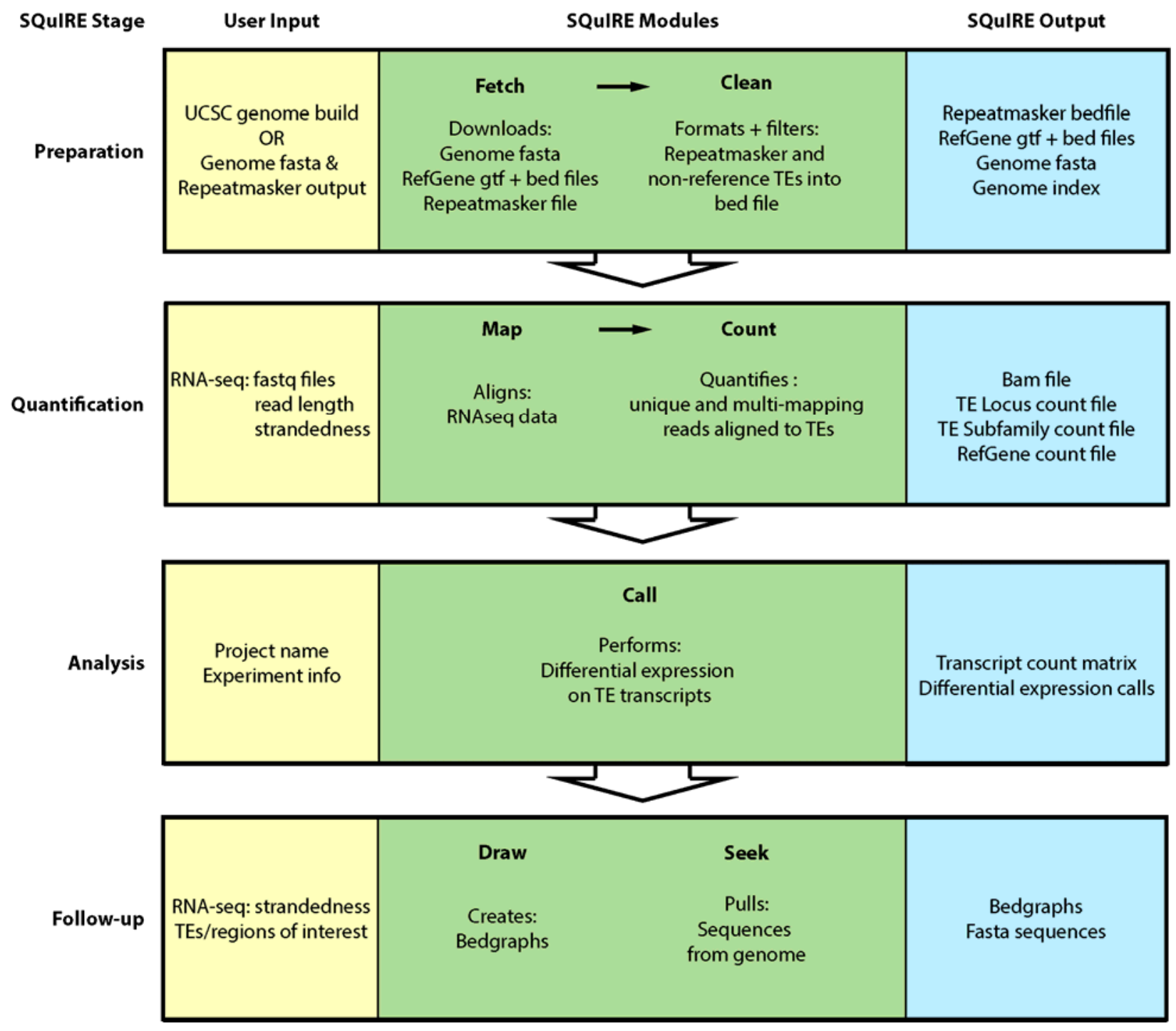

Figure 1. Schematic overview of SQuIRE pipeline. 


\section{Count Algorithm}

SQuIRE's Count algorithm addresses a fundamental issue with quantifying reads mapping to TEs:

93 shared sequence identity between TEs from the same subfamily and even superfamily. When a read

94 fragment originating from these non-unique regions is aligned back to the genome, the read may

95 ambiguously map to multiple loci ("multi-mapped reads"). This is not a major problem for older elements

96 that have acquired relatively many nucleotide substitutions, and thus give rise to primarily uniquely

97 aligning reads ("unique reads"). However, TEs from recent genomic insertions that have high sequence

98 similarity to other loci may have few distinguishing nucleotides. Among elements of approximately the

99 same age, relatively shorter TEs also have fewer sequences unique to a locus. Thus, discarding or

misattributing multi-mapped reads can result in underestimation of TE expression.

Previous TE RNA-seq analysis pipelines have been able to quantify TE expression at subfamily-level resolution. The software RepEnrich (Criscione et al. 2014) "rescued" multi-mapping reads by re-aligning

103 them to repetitive element pseudogenome assemblies of TE loci and assigning a fractional value inversely

104 proportional to the number of subfamilies to which each read aligned. These multi-mapped fractions were 105 combined with counts of unique reads aligned to each subfamily. This approach was an advance in that it

106 used information from multi-mapped reads. However, this method results in assigning fractions that are

107 proportional to the number of subfamilies that share the multi-mapped read's sequence, rather than each

108 subfamily's approximate expression level. TEtranscripts (Jin et al. 2015) expanded on this rescue method

109 by assigning an initial fractional value inversely proportional to the number of TE loci (not subfamilies)

110 to which each read aligned. This initial fractional value was then used in an expectation-maximization

111 (EM) algorithm, which iteratively re-distributes fractions of a multi-mapping read among loci (E-step) in

112 proportion to their relative multi-mapped read abundance estimated from a previous step (M-step). The

113 total of multi-mapped reads and unique reads for each loci are then summed by subfamily. However, in

114 excluding unique reads from the EM algorithm, TEtranscripts does not incorporate empirical high-

115 confidence data to infer TE expression levels from unique TE alignments. Furthermore, in calculating the 
116 relative expression level of multi-mapped reads, TEtranscripts normalizes read counts based on annotated

117 coordinates from RepeatMasker. This underestimates TE expression levels for transcripts shorter than the

118 annotated genomic length. TEtranscripts then sums the unique and multi-mapping counts for each

119 subfamily.

120 In order to accurately quantify TE RNA expression at locus resolution, Count builds on these

121 previous methods by leveraging unique read alignments to each TE to assign fractions of multi-mapping

122 reads (Fig. 2). First, Count identifies reads that map to TEs (by at least 50\% of the read length) and labels

123 them as "unique reads" or "multi-mapped reads." Second, Count assigns fractions of a read to each TE as

124 a function of the probability that the TE gave rise to that read. Uniquely aligning reads are considered

125 certain (e.g., probability $=100 \%$, count $=1$ ). Count initially assigns fractions of multi-mapping reads to

126 TEs in proportion to their relative expression as indicated by unique read alignments. In doing so, Count

127 also considers that TEs have varying uniquely alignable sequence lengths. To mitigate bias against the $n$

128 number of TEs without uniquely aligning reads, these TEs receive fractions inversely proportional to the

129 number of loci $(N)$ to which each read aligned. Then Count assigns the remainder $\left(1-\frac{n}{N}\right)$ to the TEs

130 with unique reads. To account for TEs that have fewer unique counts due to having less unique sequence,

131 Count normalizes each unique count $\left(C_{U}\right)$ to the number of individual unique read start positions, or each

132 TE's uniquely alignable length $\left(L_{U}\right)$. Among all TEs to which a multi-mapping read aligned, the TEs with

133 unique reads $(s \in T$ ) are compared with each other. A fraction of a read is assigned to each TE in

134 proportion to the contribution of the normalized unique count $\left(\frac{C_{U}}{L_{U}}\right)$ to the combined normalized unique

135 count of all of the TEs being compared $\left(\sum_{s \in T} \frac{C s}{L_{s}}\right)$. (Equation 1). The sum of unique counts and multi-

136 mapped read fractions for each TE provides an initial estimate of TE read abundance based on empirically

137 obtained unique read counts and uniquely alignable sequence.

$$
f_{T E}^{r}=\frac{\frac{c_{U}}{L_{U}}}{\sum_{s \in T} \frac{C s}{L_{S}}} \times\left(1-\frac{n}{N}\right) \quad \text { Equation } 1
$$


140 valid alignments for each read. Count next refines this initial assignment by redistributing multi-mapping

141 read fractions in proportion to estimated TE expression. To estimate expression, Count uses the a TE's

142 total read count $\left(C_{T E}=\right.$ unique read counts + multi-mapped fractions from the previous step) normalized

143 by the effective transcript length $\left(l_{T E}\right): \frac{C_{T E}}{l_{T E}}$. The effective transcript length $l_{T E}$ is calculated as the

144 estimated transcript length $L_{T E}$ subtracted by the average fragment length aligned to that TE $+1\left(l_{T E}=\right.$

$\left.145 L_{T E}-l_{\text {avg }}+1\right)$, as described previously (Li et al. 2010). All of the TEs to which a multi-mapping read

146 aligned $(s \in T)$ are compared with each other. A fraction of a read is assigned to each TE in proportion

147 to the relative normalized total count $\left(\frac{C_{T E}}{l_{T E}}\right)$ compared to the combined normalized total count of all of the

148 TEs being compared $\left(\sum_{s \in T} \frac{T_{s}}{l_{s}}\right)$, as shown in Equation 2. Count assumes this value is proportional to the

149 probability that the TE gave rise to the multi-mapping read, and assigns that fraction of a read count to the

150 TE. Because TEs with a count fraction of less than 1 have a low probability of giving rise to any read,

151 those TEs are assigned a count fraction of 0.

$$
f_{T E}^{r}=\frac{\frac{c_{T E}}{l_{T E}}}{\sum s \in T T_{S}} \quad \text { Equation } 2
$$

After the total counts (unique and multi-mapped) of each TE are re-calculated, multi-mapped reads

154 can be re-assigned in subsequent iterations of expectation (assigning multi-mapped read fractions to TEs)

155 and maximization (summation of unique and multi-mapped fraction counts). These iterations can be

156 repeated until a given iteration number set by the user or until the TE counts converge ("auto", when all

157 of the TEs with $\geq 10$ counts change by $<1 \%$ ). An example of Count output is provided in Supplemental

158 Table S1. Further details of the Count algorithm are in Supplemental Methods. 


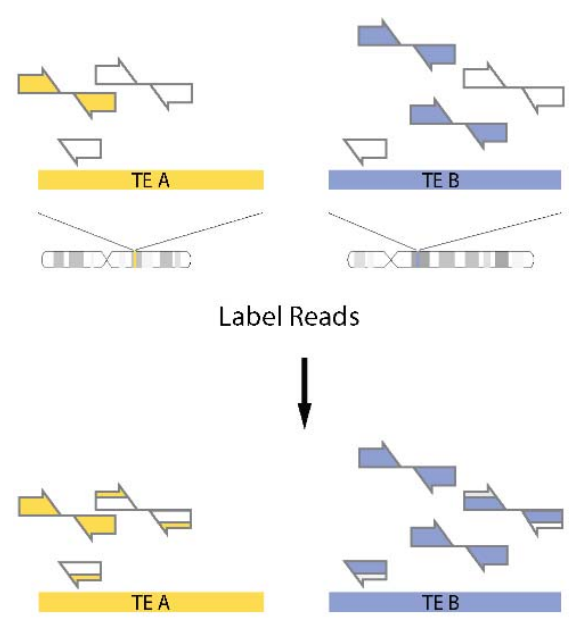

Assign

multi-mapping fragments

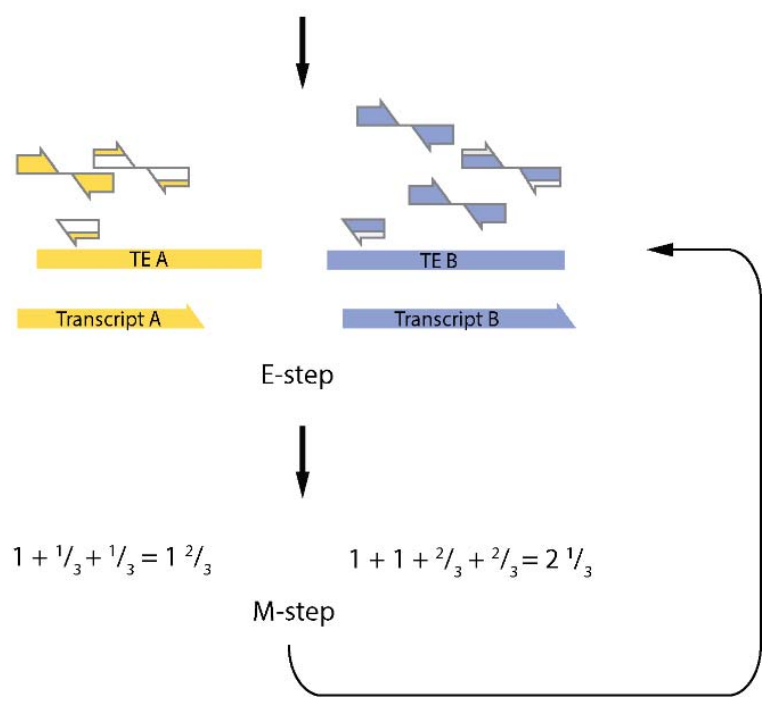

Figure 2. Schematic representation of the SQuIRE Count algorithm. First, Count labels reads as unique (filled arrows) or multi-mapping (empty arrows). Second, Count assigns fractions of multi-mapping reads in proportion to the normalized unique read expression of each TE. The partially filled arrows reflect the proportion of the read assigned to the TE of the corresponding color. Then, Count runs an ExpectationMaximization loop that estimates transcript length and reassigns multi-mapping reads for each TE (E-step), then re-estimates total read counts (M-step) until convergence. 


\section{Assessing Count Accuracy in simulated data}

To test the performance of Count, we simulated RNA-seq data from 100,000 randomly selected TEs

162 from the human GRCh38/hg38 (hg38) RepeatMasker annotation (see Methods). TEs were simulated with

163 read coverages of ranging from 2-4000X and simulated counts ranging from 2-4588. We first evaluated

164 accuracy by how closely SQuIRE Count output corresponded to the simulated read counts (i.e., \%

165 Observed/Expected). However, using this calculation is not meaningful for TEs with low simulated

166 counts: a TE with 0 counts gives an infinite value, and a reported count of 1 for a TE with 2 simulated

167 reads gives a low 50\% Observed/Expected. Thus, we were primarily interested in 'expressed' simulated

168 TEs, considering only the 99,567 TEs with at least 10 simulated reads. Second, we evaluated SQuIRE by

169 how often it correctly detected simulated TE expression (i.e., true positives) or misreported unexpressed

170 TEs (i.e., false positives).

171 To test how well SQuIRE performed leveraging only uniquely aligning read information, we first

172 evaluated the \% Observed/Expected of TE counts with 0 E-M iterations. We found that SQuIRE

173 accurately assigned read counts to most TEs, with a mean \% Observed/Expected of $98.79 \%$

174 (Supplemental Fig. S1). We predicted that this accuracy would be lower for TEs with less uniquely

175 alignable sequence. Indeed, SQuIRE was less accurate for elements with less than $10 \%$ divergence (mean

176 of $77.35 \%$ Observed/Expected). The most frequently retrotranspositionally active TEs (i.e., AluYa5,

177 AluYa8, AluYb8, AluYb9, and L1HS) had counts ranging from 48-70\% Observed/Expected, with a range

178 of 79-92\% Observed/Expected at the subfamily level (Supplemental Table S2). This illustrates that even

179 without the EM-algorithm, SQuIRE is sensitive for highly homologous subfamilies at the subfamily level.

180 Given the low recovery of simulated counts for younger elements when relying solely on uniquely

181 aligning reads, we next evaluated how much adding the EM-algorithm improved Count's performance.

182 We anticipated that the counts for most TEs would not change, but that younger elements with less

183 divergence would have improved recovery of simulated reads. Indeed, the overall \% Observed/Expected

184 counts of TE loci increased only slightly by $0.14 \%$ to a total of $98.93 \%$. However, the change in \% 
185 Observed/Expected of TEs was much greater for the most homologous active elements, improving by

$18620.47 \%$ for young Alu elements and by $21.1 \%$ for L1HS loci (Fig. 3). At the subfamily level, the \%

187 Observed/Expected of active TEs was improved by $8.1 \%$ for young Alu elements and by $2.2 \%$ for L1HS

188 (Supplemental Table S2). Using updated transcript information in the EM-algorithm is thus particularly

189 useful for TE biologists interested in younger elements that have previously been problematic to quantify

190 by RNA-seq.

191 We also wanted to evaluate SQuIRE's ability to distinguish whether a TE is expressed or not. To

192 examine how well Count detected expressed TEs, we calculated the true positive rate (TPR) as the

193 percentage of TEs with at least 10 simulated reads that SQuIRE also reported to have $\geq 10$ counts.

194 Conversely, we evaluated how often SQuIRE falsely reports TE expression by calculating the positive

195 predictive value (PPV) as the percentage of TEs with $\geq 10$ reported counts that were in fact simulated to

196 have $\geq 10$ reads. The true negative rate, or how often SQuIRE correctly reports that a TE is not expressed,

197 is less informative for evaluating TE estimation accuracy because the number of TEs in the hg38 genome

198 is so high (>4 million TEs) that the true negative value would outweigh the false positive value (Saito and

199 Rehmsmeier 2015). Overall, SQuIRE had both a high TPR of 98.5\% and high PPV of 99.4\%. These

200 values were lower for frequently retrotranspositionally active Alus (TPR=68.75-83.33\%, PPV=64.29-

$201100 \%$ ) and L1HS (TPR=100\%, PPV=62.86\%) using only unique reads for TE expression estimation

202 (Supplemental Table S3). However, using the EM algorithm improved the TPR for Alus (TPR=85.22\%-

$203100 \%$ ) by reducing false negative reports and the PPV for L1HS (PPV=78.57\%) by reducing false

204 positives. 


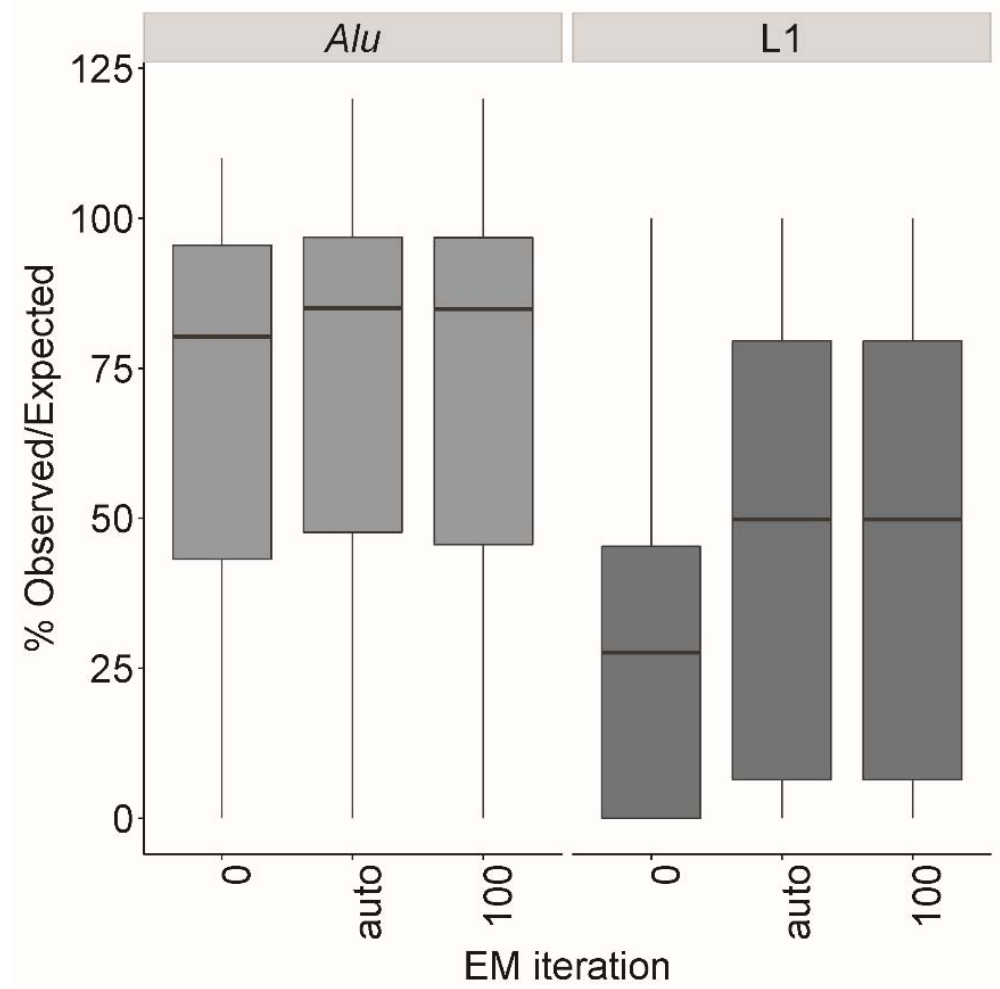

Figure 3. Running EM iterations improves the \% Observed/Expected for SQuIRE

Count for the frequently retrotranspositionally active Alu (AluYa5, AluYa8, AluYb8,

Alu $\mathrm{Yb9}$ ) and L1 (L1HS) subfamilies compared to no EM iterations (i=0), and does not degrade with increasing iterations (i=100). By default (i="auto"), SQuIRE Count continues the EM-algorithm until each TE with more than 10 reported read counts changes by less than $1 \%$. 


\section{Endogenous LINE-1 detection with Count}

To assess Count's ability to detect endogenous LINE-1 expression using biological data, we

209 evaluated the expression level of L1 at loci previously characterized by other methods. Because L1s often

210 become 5' truncated upon insertion (Perepelitsa-Belancio and Deininger 2003), Deininger et al.

211 performed 5' rapid amplification of cDNA ends (RACE) on cytoplasmic HEK293 RNA to enrich for full-

212 length L1 RNA. They also performed RNA-seq on polyA-selected cytoplasmic HEK293 RNA to identify

213 L1 loci that have downstream polyadenylation signal. We filtered their findings for L1 loci that had $>5$

214 mapped RNA-seq reads from both 5'RACE and poly-A selected RNA libraries (Deininger et al. 2017) to

215 compare with SQuIRE. We then examined the expression reported by SQuIRE at these 33 loci in paired-

216 end, total RNA from HEK293T cells (GSE113960). We found that 31 (93.4\%) had > 10 SQuIRE read

217 counts, confirming their expression (Supplemental Table S4). This suggests that Count can detect L1

218 expression in RNA-seq libraries that are not enriched for L1 loci.

219 Only a subset of the L1s evaluated by Deininger et al. belonged to L1HS, the youngest family of L1s.

220 Because L1HS loci can be retrotranspositionally active, they can generate insertions that are

221 polymorphic or novel compared to the the reference human RepeatMasker annotation. Reads from TE

222 insertions that are not present in the RepeatMasker annotation can be misattributed to unexpressed, fixed

223 TEs, which can result in "false positive" reports of expression at silent loci. To test how this affects

224 Count, we transfected HEK293T cells with an empty pCEP4 plasmid or with a plasmid containing L1RP,

225 an L1HS with known retrotransposition activity (Schwahn et al. 1998; Kimberland et al. 1999). The

226 transfection of L1RP resulted in increased L1HS-aligning reads (254,681 reads) compared to L1HS loci

227 in L1RP-negative cells (2,671 reads) (Supplemental Fig. S2). The differences in L1HS expression in

228 L1RP-transfected cells was higher than what we would expect from endogenous, polymorphic insertions

229 based on previous estimates of polymorphic and fixed L1HS expression in HEK293T cells using unique

230 reads within $1 \mathrm{~kb}$ downstream of L1HS loci (Philippe et al. 2016). Because Philippe et al. suggested that

231 polymorphic L1HS insertions were transcribed at levels similar to fixed full-length L1HS loci, we sought 
232 to mimic polymorphic L1HS expression levels more consistent with previously reported levels. To

233 determine comparable fixed L1HS expression levels in our control HEK293T RNA-seq data, we

234 examined the Count output at loci with reported expression by Phillipe et al. (145 read counts). We then

235 downsampled the L1RP-aligning reads from L1RP transfected HEK293T cells to a similar number (153

236 reads). To simulate a range of polymorphic L1HS expression levels, we also downsampled RNA-seq

237 reads that aligned to the L1RP plasmid to $2 \mathrm{X}$ and 20X the fixed active L1HS expression level (302 and

238 3,091 reads). For these downsampled reads, we identified their other, off-target alignments to the

239 reference genome. To control for potential biological effects of L1RP transfection on TE counts, we

240 'spiked in’ these downsampled reads from L1RP-transfected cells into RNA-seq data from HEK293T

241 cells transfected with an empty pCEP4 plasmid. We then calculated the number of false positive L1 loci

242 that became 'expressed' with > 10 counts after the in silico spike-in. We focused on the 3 youngest L1

243 subfamilies that share the greatest homology with the L1RP sequence (i.e., L1HS or L1PA1, L1PA2, and

244 L1PA3) (Smit et al. 1995; Boissinot et al. 2000; Lee et al. 2007) and compared their false positive rates to

245 older L1 loci (Fig. 4). When the alignments of 153 reads were spiked in, we found that the false positive

246 rate (FPR) of the youngest L1 subfamilies were comparable to each other, ranging from 34-38\%.

247 However, as the spiked in alignments increased to 302 and 3091 reads, the FPR increased for L1HS to

$24850.68 \%$ but not the other subfamilies. This indicates that polymorphic L1HS expression primarily affects

249 the alignments to L1HS loci, and not the loci of closely related subfamilies.

250 L1-mapping methods (Upton et al. 2015; Rodić et al. 2015; Iskow et al. 2010; Ewing et al. 2010) and

251 TE insertion detection software for whole genome sequencing (Gardner et al. 2017; Lee et al. 2012;

252 Keane et al. 2013; Stewart et al. 2011; Sudmant et al. 2015; Ewing et al. 2011) can identify locations of

253 non-reference TE insertions. Validating these insertions by PCR and Sanger sequencing can provide not

254 only unique sequence flanking the insertion but potentially also the TE sequence. Users can input a

255 custom table to SQuIRE Map and Clean (Supplemental Table S5) to add non-reference TEs and their

256 flanking sequence to the alignment index and RepeatMasker BED file. We evaluated how incorporating 

aCC-BY-NC 4.0 International license.

257 the non-reference table containing information about the L1RP plasmid affected the FPR in HEK293T

258 cell data. We found that the FPR for L1HS only increased from 36.67\% with 153 reads spiked in to

$25939.34 \%$ with 3091 reads spiked in. Thus, adding L1RP information improved Count's accuracy at higher

260 L1RP in silico expression levels. 


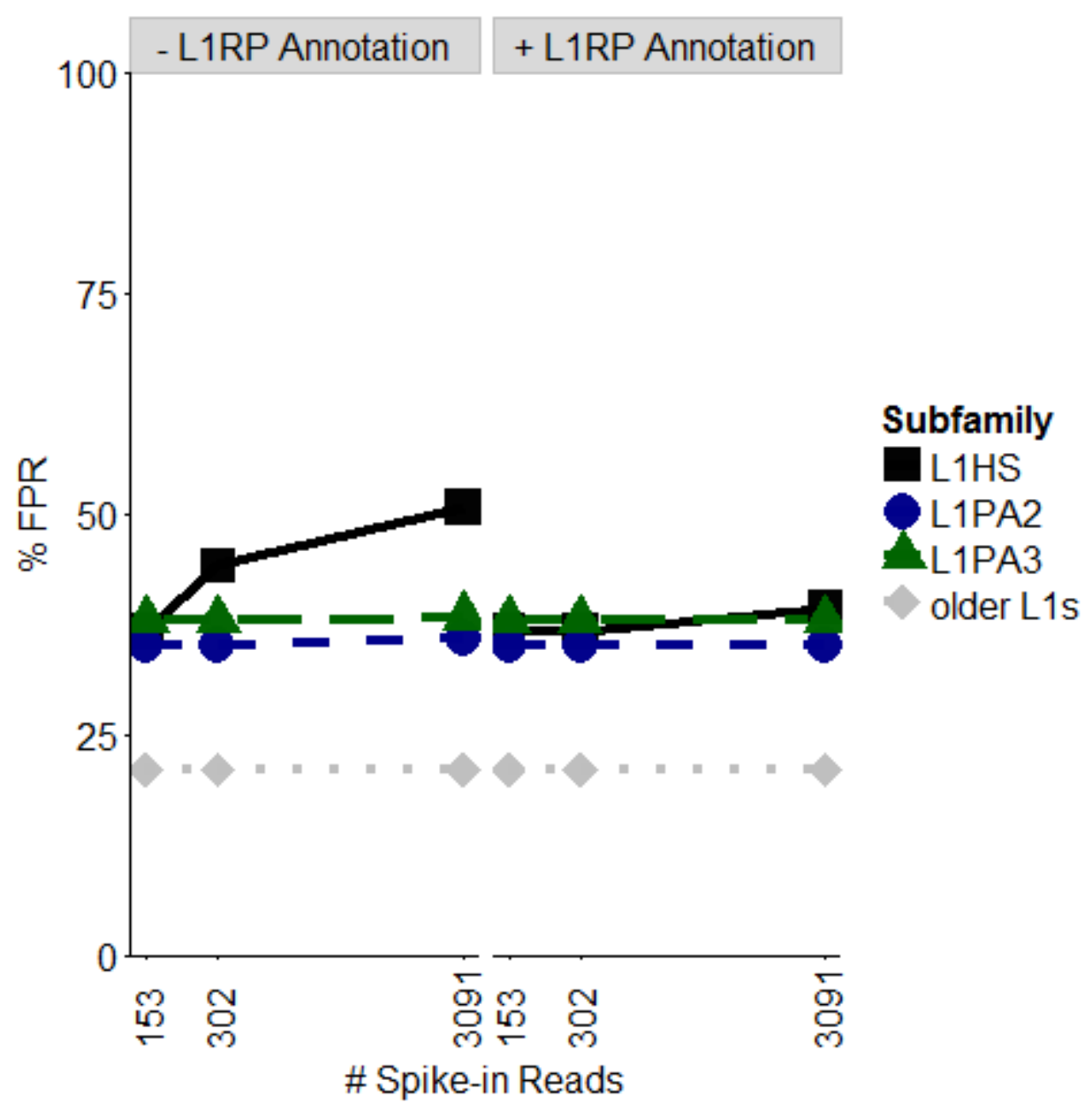

Figure 4. False positive rate (FPR) of L1 loci expression in HEK293T cells when spiking in L1RP-aligning reads. False positive expression is implicated a locus that previously had $<10$ reads has $\geq 10$ reads after spike-in. \% FPR is the percentage of loci with false positive loci relative to the total number of loci with $\geq 10$ SQuIRE read counts. The number of spike-in reads $(153,302,3091)$ represents $1 \mathrm{X}, 2 \mathrm{X}$ and 20X predicted endogenous polymorphic L1HS expression levels based on findings from Phillipe et al. 2016. The FPR is robust for older L1 subfamilies with increased spike-in reads. The addition of L1RP annotation in a non-reference table reduces the change in false positive rate for L1HS after increasing spike-in reads. 


\section{Comparison to other software}

Currently published TE analysis software include RepEnrich, TEtranscripts, and TETools

264 (Criscione et al. 2014; Jin et al. 2015; Lerat et al. 2016). We used the simulated hg38 TE data described

265 above to compare the recovery of simulated reads to the correct subfamily among TE quantification

266 software (\% Observed/Expected). For mapping, we ran each software's recommended aligner: STAR

267 (used by SQuIRE and TEtranscripts), Bowtie 2 (used by TETools), and Bowtie 1 (used by RepEnrich).

268 We found that SQuIRE $(99.86 \% \pm 1.46 \%)$, TETools $(100.14 \pm 2.21 \%)$, and TEtranscripts $(95.89 \pm$

$26916.41 \%$ ) had comparable \% Observed/Expected rates (Supplemental Fig. S3). In contrast, RepEnrich

$270(108.77 \pm 40.67 \%)$ was less accurate in terms of $\%$ Observed/Expected. This is likely attributable to

271 RepEnrich's recommended use of Bowtie 1, which discards discordant reads and limits the number of

272 attempts to align both paired-end mates to repetitive regions. To support this, we compared how often

273 each aligner mapped a uniquely aligning simulated read to the correct location. We indeed found that

274 Bowtie 1 failed to report unique reads more often in a paired-end library compared to single-end

275 (Supplemental Table S6).

276 To compare SQuIRE to other TE analysis tools with biological data, we ran each pipeline on

277 publically available adult C57Bl/6 mouse tissue RNA-seq data (Brawand et al. 2011) using

278 GRCm38/mm10 (mm10) TE annotation. We compared the expression of subfamilies in testis compared

279 to pooled data from brain, heart, kidney, and liver tissues. To independently evaluate the fold-changes of

280 TE RNA between testis and somatic tissues, we also used our previously published adult C57B1/6 mouse

281 Nanostring results (Gnanakkan et al. 2013). Unlike RNA-seq analysis, which infers transcript levels by

282 counting reads, Nanostring uses uniquely mapping probes to capture and count RNA molecules. We

283 compared the Nanostring $\log _{2}$ fold changes $\left(\log _{2} \mathrm{FC}\right)$ of TE subfamily expression in testis and pooled

284 somatic tissue to the $\log _{2}$ FC values found by SQuIRE, RepEnrich, TEtranscripts, and TETools

285 (Supplemental Fig. S4). We first looked at how often the direction of fold change corresponded between

286 each tool and Nanostring. For the 16 subfamilies queried, SQuIRE and TETools shared the same direction 
of fold change as Nanostring more often than the other tools (SQuIRE: 12, TETools: 12, TEtranscripts: 9 , RepEnrich: 8). Moreover, compared to TETools, SQuIRE reported $\log 2 \mathrm{FC}$ values closer to the expected values from Nanostring (mean absolute differences in log2FC from Nanostring- SQuIRE: 0.965, TETools: 1.34, TEtranscripts: 1.16, RepEnrich: 1.11). subfamilies analyzed by Nanostring and the TE analysis tools, we found that the reported subfamily-level expression could be attributed to fewer than $7 \%$ of each subfamily's loci (Supplemental Fig. S5). This suggests that regulation of TE transcription is not necessarily shared across all TEs from the same subfamily. On the other hand, whereas the other subfamilies studied by Nanostring have only 1-4 significantly differentially expressed $\operatorname{loci}(\log 2 \mathrm{FC}>1$, padj $<0.05)$, the IAPLTR3 subfamily has 11 loci

297 that are all differentially expressed in testis compared to somatic tissues (Fig. 5A). To test whether this was an enrichment relative to the representation of IAPLTR3 in the mouse genome, we performed a Fisher's exact test and found that IAPLTR3 loci were 10-fold more likely than expected to be differentially expressed in testis (OR: 10.56, 95\%CI: 5.25-18.97, padj < 1.61 e-08). This suggests that a

301 subset of TE locus expression may still be impacted by subfamily-specific regulation. context of these 3 loci, we found that all were located within genes with known broad tissue expression

307 (Gpbp1, Csnk2a1, Kyat1, respectively) (Yue et al. 2014), with examples shown in Supplemental Figure

308 S6. Another locus from the MURVY subfamily is in a cluster of TEs exhibiting high testis-restricted

309 expression. In examining the transcript overlapping the MURVY locus, we see that the transcript initiates 310 outside of the locus and find that the transcript is an alternative splicing isoform with splice donors from 311 the third and fourth exons of a gene $\sim 5 \mathrm{~kb}$ away (Fig. 5B). The gene, Gm11981, is a long noncoding RNA 
312 (lncRNA) known to exhibit testis-restricted expression (Yue et al. 2014). The different MURVY-

313 containing transcripts illustrate how the relationship between TE expression and neighboring transcription

314 can vary across loci from the same subfamily. We also examined ERVB4-1B and IAPLTR3, the two LTR

315 retrotransposon subfamilies that exhibited the highest fold change by Nanostring. These subfamilies were

316 represented in the high-expressing, tissue-restricted loci cluster (Fig. 5A). While the transcription of the

317 ERVB4-1B locus on chr13 did not extend beyond annotations for that subfamily (Fig. 5C), the IAPLTR3

318 loci on chr14 (Fig. 5D) and chr18 are part of longer transcripts that initiate outside of the annotated TE.

319 Unlike the MURVY locus on chr11, there is no evidence of splicing into the IAPLTR and ERVB4-1B

320 loci. Thus, TEs from different subfamilies may be subject to different mechanisms of transcriptional

321 regulation as evidenced by expression within different transcript structures. Altogether, this stresses the

322 utility of using SQuIRE to analyze TE transcription at the locus level. 


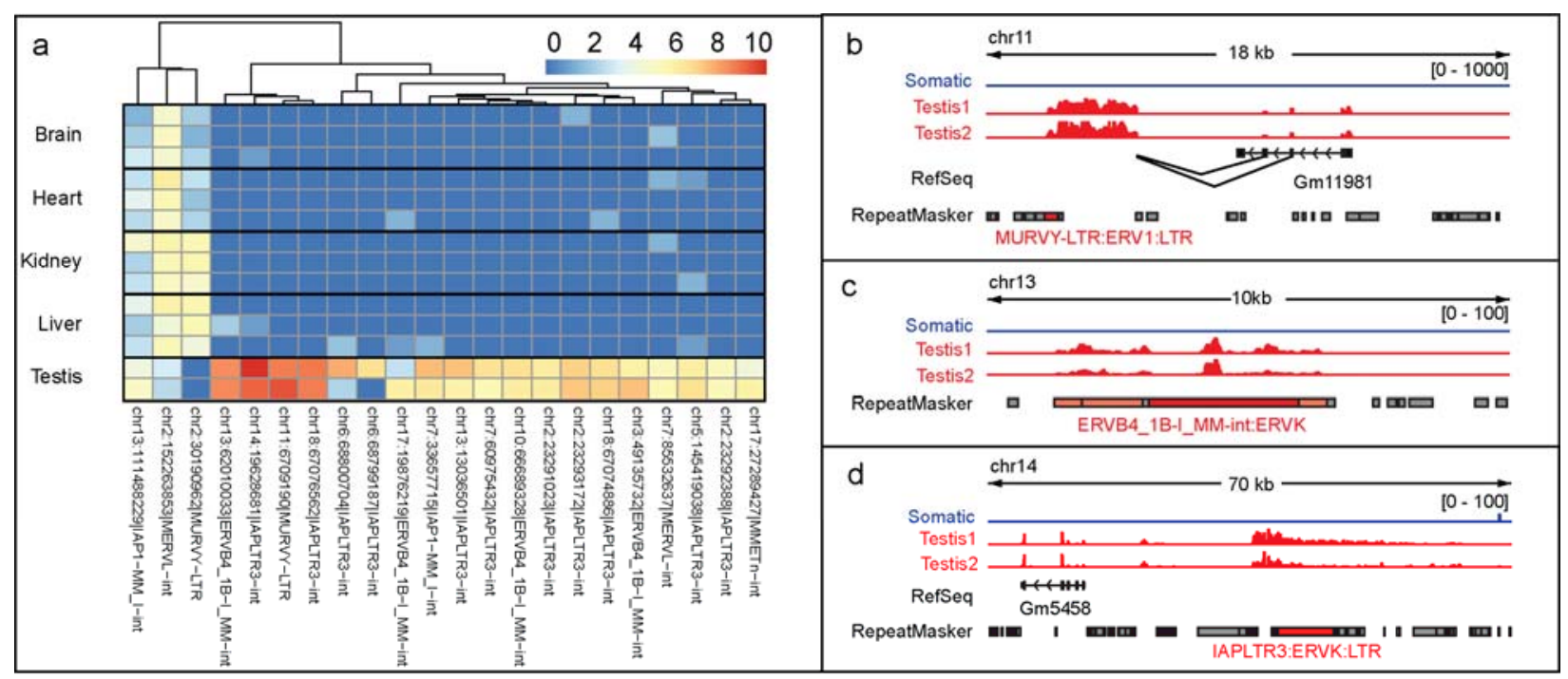

Figure 5. Differentially expressed TE loci belonging to subfamilies previously analyzed by Nanostring a. The $\mathrm{X}$-axis represents replicates of somatic and testis tissue samples from adult C57B1/6 mouse. The Y-axis represents differentially expressed TE loci. The heatmap colors represent the $\log 2$ of total read counts +1 for each TE locus. b-d. Examples of intergenic TE loci differentially expressed in testis compared to somatic tissues. Tracks from brain, heart, kidney and liver replicates were collapsed into a single track. The scales of count expression are shown in brackets. The RefSeq track represents annotated genes. The RepeatMasker track represents transposable elements annotated in the reference genome. Transposable elements colored in red belong to the subfamily indicated; dark red indicates that that RepeatMasker entry meets significant differential expression thresholds $(\log 2 \mathrm{FC}>2$, padj < 0.05$)$. 


\section{Benchmarking for SQuIRE's Memory Usage and Running Time}

To benchmark SQuIRE's memory usage and running time for RNA-seq data of different

328 sequencing depths, we subset the high-depth (mean 263 million reads across 8 lanes) HEK293T cell line

329 RNA-seq data into 1, 2, and 3-lane libraries with a mean sequencing depth of 32, 65, and 98 million

330 reads. We evaluated the speed and memory performance of each Quantification and Analysis stage tool

331 for each sequencing depth (Fig. 6) using 8 parallel threads and $64 \mathrm{~Gb}$ of available memory. We found that

332 sequencing depth had the greatest effect on Count, taking 8.6 hours to complete the 3-lane library

333 compared to 2.4 hours for the 1 lane library. The other tools took much less time and were less affected

334 by sequencing depth. Map took 1-2 hours for the different libraries. Call running time was also

335 independent of library size, but it was greater when including all TE counts (10 minutes) compared to

336 subfamily counts ( 2 minutes). We found that the memory usage of each tool was largely independent of

337 sequencing depth, taking between 39-40Gb of Memory for Map, 30-32Gb for Count, and 7-8Gb for

338 Call.

$339 \quad$ Implementation

340 Our efforts at making SQuIRE easy to use has resulted in a simple installation process in which the

341 user can copy and paste lines of code to install all prerequisite software and set up SQuIRE (Table 1). In

342 addition, SQuIRE is the only program that downloads reference annotation for assembled genomes

343 available on UCSC, allowing it to be easily adaptable to a variety of species. For genomes from non-

344 model organisms or organism strains with high divergence from the reference annotation, SQuIRE can

345 also use RepeatMasker software output for even wider compatibility. To ensure that the pipeline is

346 streamlined and that the outputs are reproducible, SQuIRE also implements alignment and differential

347 expression for the user. In making SQuIRE as user-friendly as possible, we intend to improve the

348 reproducibility of bioinformatics in the TE field. 



Figure 6. Usage data for the main modules of SQuIRE. Time (Hours)

and Memory for SQuIRE Count, Map and Call. Mean library sizes for

RNA seq data were 1 lane $=32,912,528$ reads, 2 lanes $=65,573,850$ reads,

3 lanes $=98,757,439$ reads. 


\begin{tabular}{|l|l|l|l|l|}
\hline & SQuIRE & RepEnrich & TEtranscripts & TETools \\
\hline $\begin{array}{l}\text { Provides Locus-level TE } \\
\text { RNA quantification }\end{array}$ & YES & -- & -- & -- \\
\hline $\begin{array}{l}\text { Provides TE transcript } \\
\text { information }\end{array}$ & YES & -- & -- & -- \\
\hline $\begin{array}{l}\text { Copy-and-paste } \\
\text { installation }\end{array}$ & YES & -- & -- & -- \\
\hline $\begin{array}{l}\text { Provides prerequisite } \\
\text { annotation files for any } \\
\text { species }\end{array}$ & YES & -- & -- & -- \\
\hline $\begin{array}{l}\text { Can incorporate non- } \\
\text { reference TEs }\end{array}$ & YES & -- & -- & YES \\
\hline $\begin{array}{l}\text { Performs alignment } \\
\text { STAR }\end{array}$ & $\begin{array}{l}\text { Recommends } \\
\text { Bowtie 1 }\end{array}$ & $\begin{array}{l}\text { Recommends } \\
\text { STAR }\end{array}$ & $\begin{array}{l}\text { YES - uses } \\
\text { Bowtie 1 or } \\
\text { Bowtie 2 }\end{array}$ \\
\hline $\begin{array}{l}\text { Uses genome for } \\
\text { alignment }\end{array}$ & YES & $\begin{array}{l}\text { YES - Genome + } \\
\text { TE } \\
\text { pseudogenome }\end{array}$ & YES & -- \\
\hline $\begin{array}{l}\text { Provides gene expression } \\
\text { quantification }\end{array}$ & YES & -- & YES & -- \\
\hline $\begin{array}{l}\text { Performs differential } \\
\text { expression }\end{array}$ & YES & -- & YES & YES \\
\hline
\end{tabular}

Table 1. Feature comparison of RNA-seq Analysis tools for TEs. 


\section{Discussion}

We have developed Software for Quantifying Interspersed Repeat Expression (SQuIRE) to

353 characterize TE expression using RNA-seq data. TEs are highly repeated in the genome, which can pose

354 challenges for mapping reads unambiguously to specific transcribed loci. SQuIRE is the first RNA-seq

355 analysis software that provides locus-specific TE expression quantification while also outputting

356 subfamily-level expression estimates (Table 1). Our approach uses unambiguously mapping reads and an

357 Expectation-Maximization algorithm to estimate levels of TE transcripts. SQuIRE additionally provides

358 information on the structure of each TE transcript, which can be shorter or longer, sense or antisense

359 compared to the annotated repeat. We have shown that SQuRE can correctly attribute a high percentage

360 of reads originating from TEs using simulated data. Although this percentage is lower for frequently

361 retrotranspositionally active, less divergent TEs (e.g., AluYa5, AluYa8, AluYb8, AluYb9, L1HS), we

362 found that implementation of an Expectation-Maximization (EM) algorithm (Jin et al. 2015; Li and

363 Dewey 2011) improves accuracy and lowers both false positive and false negative estimations of whether

364 a TE is expressed. This finding also holds in biological settings, where SQuIRE is able to correctly

365 identify instances of full-length L1 expression in total RNA RNA-seq data from cell lines wherein

366 previous studies had identified these loci using a combination of 5'RACE and 3' primer extension

367 methods (Deininger et al. 2017). This confirms that SQuIRE can detect the expression of TEs in the

368 reference genome that have in the past been problematic for global TE RNA expression analysis.

369 The ongoing activity of TEs also results in a significant number of mobile element insertion variants

370 (MEI) (Beck et al. 2010; Sudmant et al. 2015; Stewart et al. 2011). Numerous commonly occurring

371 structural variants owed to retrotransposition are missing in reference genome assemblies. SQuIRE

372 provides users with two options to query transcription of these repeats. First, it can detect their

373 transcription at the subfamily level. We have shown that SQuIRE can detect expression of L1HS elements

374 when we express an ectopic sequence. It maintains a low false positive rate of misattributing these reads

375 to endogenous L1HS loci. Thus, SQuIRE can be useful for detecting altered regulation of young TE 
376 subfamilies even when specific loci that are expressed are unknown. Secondly, SQuIRE can use

377 sequences of known, non-reference TE insertion polymorphisms to detect locus-specific expression when

378 these are available. For example, in the human genome, L1HS element sites and sequences can be

379 obtained by targeted TE insertion mapping (Upton et al. 2015; Rodić et al. 2015; Iskow et al. 2010;

380 Ewing et al. 2010) or whole genome sequencing (Gardner et al. 2017; Lee et al. 2012; Keane et al. 2013;

381 Ewing et al. 2011). Polymorphic TE insertions have been reported to databases such as euL1db (Mir et al.

382 2015), dbRIP (Wang et al. 2006) and 1000 Genomes Project (Sudmant et al. 2015). If the polymorphic

383 insertions have been verified and sequenced in the user's samples, SQuIRE is capable of incorporating

384 user-provided, non-reference TE sequence to estimate TE expression at these loci. This may be a useful

385 feature for understanding functional consequences of these insertion variants (Payer et al. 2017).

The SQuIRE algorithm builds on strategies used by previous TE analysis software (Criscione et al.

387 2014; Jin et al. 2015; Lerat et al. 2016). Here, we show that SQuIRE provides additional features and

388 improves on the accuracy of these methods, as assessed using both simulated reads and orthogonal

389 approaches to measure $\log _{2}$ fold changes in mouse tissue comparisons. Our findings suggest that

390 important biologic insights can be gained by examining TE transcription at the locus level.

391 To date, locus-specific studies of TE expression and activity have mostly focused on identifying

392 transcriptionally and retrotranspositionally active L1s in the human genome (Deininger et al. 2017;

393 Philippe et al. 2016; Scott et al. 2016; Brouha et al. 2003; Beck et al. 2010; Tubio et al. 2014; Pitkänen et

394 al. 2014). In applying SQuIRE to study locus-specific TE expression genome-wide in mouse tissues, we

395 can see that this paradigm is not unique to L1s or humans. It seems a very limited subset of TE loci are

396 transcribed with complex patterns of tissue-specific expression. Furthermore, we found that the tissue

397 expression patterns of TE loci were driven by a variety of transcriptome contexts: broadly expressed

398 mRNA transcripts, testis-specific lncRNA and authentic TE 'unit' transcripts. How these TEs affect

399 genome regulation remains an open question. Prior to SQuIRE, the inability to map TE expression limited

400 genome-wide analysis of TEs to the effects of cis-acting elements on transcriptional (Faulkner et al. 

aCC-BY-NC 4.0 International license.

401 2009; Kalitsis and Saffery 2009; Le et al. 2015; Xie et al. 2013) and post-transcriptional (Stower 2013;

402 Sorek et al. 2002; Ecco et al. 2016; Athanasiadis et al. 2004) regulation. Further, the effects of

403 neighboring genes on TE transcription are not well-understood. In providing locus-level TE transcript

404 estimations, SQuIRE can enable studies that dissect the regulatory impacts of TE and gene expression. 


\section{Methods}

\section{Implementation of STAR aligner in Map}

Map uses parameters tailored to the alignment of TEs. By default STAR only reports reads that map

410 paired-end reads, Map also reports paired reads that map discordantly (--chimSegmentMin

411 <read_length>) and single reads with unmapped mates (--outFilterScoreMinOverLread 0.4 -

412 outFilterMatchNminOverLread 0.4). Map can incorporate the non-reference TE sequences and generate a

413 FASTA file that STAR adds to the genome index with the option "- - genomeFastaFiles <fasta> ". To

414 provide splicing information to the tools in the Analysis Stage, Map also uses the UCSC RefSeq gene

415 annotation and assesses reads overlapping splice junctions with the options “- - sjdbGTFfile <gtf> --

416 sjdbOverhang <read_length -1> --twopassMode Basic”. Map produces a sorted BAM file that includes

417 intron and splicing information for downstream transcriptome assembly analysis.

\section{$418 \quad$ Implementation of StringTie in Count}

419 Count runs StringTie (Pertea et al. 2015)using these default settings guided by RefSeq gtf obtained 420 from UCSC with Fetch. Count uses the “-e" StringTie option to quantify expression only to annotated 421 transcripts without assembly of novel transcripts. We convert the fpkm values to counts by multiplying 422 the per-exon coverage by exon length normalized by read length.

\section{DESeq2 Implementation in Call}

424 Call incorporates the Bioconductor package DESeq2 (Love et al. 2014; Huber et al. 2015) with its

425 suggested parameters. Users input the sample names and experimental design (ie which samples are 426 treatment or control), which Call uses to find Count data and create a count matrix for annotated RefSeq 427 genes, StringTie transcripts and TEs. Call outputs differential expression tables and generates MA-plots, 428 data quality assessment plots, and volcano plots. 
To visualize the distribution of reads across the TE, Draw runs STAR (Dobin et al. 2013)with the

431 parameters “-runMode input AlignmentsFromBAM -outWigType bedGraph" to provide visualization of

432 read alignments. It will output bedgraphs of all reads ("multi”) and only uniquely ("unique”) aligning

433 reads. Draw also compresses the bedgraphs into bigwig format for IGV (Robinson et al. 2011) and UCSC

434 Genome Browser (Rosenbloom et al. 2014) viewing. If the RNA-seq data is stranded it will output unique 435 and multi bedgraphs for each strand.

\section{RNA-seq simulation}

We randomly selected 100,000 TEs from the hg38 Repeatmasker annotation downloaded by Fetch.

438 We limited our list of potential TEs to those included in TEtranscripts (Jin et al. 2015) and RepEnrich

439 (Criscione et al. 2014) to enable comparisons between these different programs. Using the selected TE

440 coordinates we generated a BED file using Clean and obtained Fasta sequences using Seek. From these

441 TE sequences, we used the Polyester package from Bioconductor ( $\mathrm{R}$ version 3.4.1, Huber et al. 2015)

442 (Huber et al. 2015)to simulate 100bp, paired-end, stranded RNA-seq reads with normally distributed

443 fragment lengths around a mean of $250 \mathrm{bp}$. We simulated a uniformly distributed sequencing error rate of

$4440.5 \%$. TEs were simulated with a mean read coverage of $20 \mathrm{X}$, with 250 TEs deviating from that mean

445 between 2-100 fold.

\section{HEK293T Cell Culture, Transfection and Sequencing}

Tet-On HEK293TLD (293T) cells (Taylor et al. 2013) were grown at 37C, 5\% CO2 in DMEM with

448 10\% Tet-Free FBS (Takara, Mountain View, CA) and passaged every 3-5 days as needed.

449 LINE expression constructs were cloned into the pCEP4 backbone (Thermo Fisher Scientific,

450 Waltham, MA) modified to confer puromycin resistance. Plasmids encoded either L1RP (MT302) or had

451 no insert (Taylor et al. 2013). For transfection, 300,000 293T cells were plated in 2 mL volume. 24 hours

452 later, cells were transfected using a cocktail of 2 ug plasmid DNA and 6 uL Fugene HD (Promega), and 
453 puromycin was added 24 hours later for a total of 3 days of selection. 500,000 cells were then plated in 3

454 wells each, and doxycycline was added 2 hours later (final concentration of $1 \mathrm{ug} / \mathrm{ml}$ ) to induce $\mathrm{L} 1$

455 expression. RNA was collected after 72 hours of L1 expression using the Zymo Quick-RNA MiniPrep kit

456 (Zymo Research, Tustin, CA). The RNA libraries of transfected 293T cells were prepared using the

457 Illumina TruSeq Stranded Total Library Prep Kit with Ribo-Zero Gold (San Diego, CA) to provide

458 stranded, ribosomal RNA depleted RNA. The libraries were sequenced on an Illumina HiSeq 2500, using

4596 samples per lane across 8 lanes with paired-end 100bp reads. We generated a mean of 263,127,067

460 paired reads per sample. The raw sequencing data were deposited to the NCBI Genome Expression

461 Omnibus (GEO) with accession number GSE113960.

For detection of fixed L1 expression identified by Deininger et al. by 5'RACE and poly-A selected

464 RNA sequencing in HEK293 cells, we ran SQuIRE Map, Count, and Call on HEK293T cell samples

465 transfected with empty L1RP vector (DA5 and DA6). To determine the effect of L1RP transfection on the

466 false positive rate of L1 RNA estimation, we ran Map and Count on HEK293T cells transfected with

467 L1RP and vector. To simulate the effect of polymorphic TE expression on typical RNA-seq samples, we

468 downsampled a transfected (DA1) and control (DA5) sample to a single lane per sample (average 32

469 million reads). To identify L1RP aligning reads in the L1RP-transfected cell, we used SAMtools (Li et al.

4702009 ) to identify reads that align to the chromosome construct provided by the non-reference table

471 (Supplemental Table S5). To downsample the L1RP-aligning reads, we used the SAMtools “-s

$472<$ INT.FRAC> " option with $0.01,1.001$, and 3.0004 as inputs. The integer before the decimal indicates

473 the seed value and the number after the decimal indicates the fraction of total alignments desired for

474 subsampling. We then identified all alignments to the genome sharing the same Read IDs as the down-

475 sampled L1RP-aligning reads. We used SAMtools merge to combine the alignments of L1RP-aligning

476 reads with the BAM file of the HEK293T cell sample transfected with empty vector (DA5).

\section{TE RNA-seq tool Comparison}


Adult C57BL/6 mouse RNA-seq data were obtained from GEO with accession number GSE30352.

All pipelines were run on a server with a maximum of 128 GB memory available and 8 threads (-p

480 setting).

RepEnrich (Criscione et al. 2014)- We obtained the hg38 annotation for RepeatMasker from the

482 RepEnrich GitHub website. For the mm10 annotation, we obtained the mm10.fa.out.gz RepeatMasker

483 (Smit, AFA, Hubley, R \& Green) annotation from the RepeatMasker website. We ran the setup for

484 RepEnrich following instructions from the website for each genome build. We then mapped the data to

485 the genome using Bowtie 1 (Langmead et al. 2009) according to RepEnrich's instructions to generate

486 separate uniquely mapping sam and multi-mapping read fastq files. These were then used for the

487 RepEnrich software with the "-pairedend TRUE" parameter for simulated human data, and "—pairedend

488 FALSE" for mouse data.

TETools (Lerat et al. 2016) - We generated rosette files for hg38 and mm10 for TETools by taking

490 the Repeatmasker annotation from Clean for the first column and the repeat taxonomy for the second

491 column (subfamily:family:superfamily). We used the BED file from Clean with Seek to obtain TE

492 FASTA sequences for generation of a pseudogenome for TETools. TETools was run with the "-bowtie2",

493 “-RNApair" and "-insert 250" parameters for simulated human data and "-bowtie2","-insert 76" for

494 mouse data. website. We aligned the data to the genome with STAR using “--winAnchorMultimapNmax 100","--

497 outFilterMultimapNmax 100" parameters for multi-mapping. We then ran TEtranscripts with the “--mode 498 multi" setting to utilize its expectation-maximization algorithm for assigning multi-reads for the resulting 499 SAM file. Since TEtranscripts analyzes TE and gene expression together, we used refGene annotation 500 obtained by SQuIRE Fetch for the required gtf file. We used the parameters “--format SAM”, “--mode 501 multi”, “--stranded yes” for simulated human data, and "--format SAM”, “--mode multi”, “--stranded no" 502 for mouse data. 


\section{Aligner Comparison}

504 We ran the aligners Bowtie1 (Langmead et al. 2009), Bowtie2 (Langmead and Salzberg 2012), and

505 STAR (Dobin et al. 2013) on the simulated TE RNA-seq data described above. We set each aligner to

506 output a maximum of 2 valid alignments to quickly identify uniquely aligning reads with the parameter "-

507 m2” for Bowtie 1, “-k2” for Bowtie 2, and “--outSAMmultNmax 2” for STAR. We also ran STAR with

508 the parameters “--outFilterScoreMinOverLread 0.4 --outFilterMatchNminOverLread 0.4 --

509 chimSegmentMin 100" to allow for discordant alignments, which STAR excludes by default. Bowtie2

510 reports discordant alignments by default, while Bowtie 1 can only report paired alignments. We used

511 BEDTools (Quinlan and Hall 2010) to intersect the BAM outputs to RepeatMasker annotation to identify

512 the TEs to which the aligners mapped the reads. Reads that only appeared once as "uniquely aligning".

513 We assessed whether the mapped TE matched the templating TE for the simulated read to determine if

514 the uniquely aligning reads mapped to the correct location.

\section{$515 \quad$ Data Access}

516 The raw sequencing data and SQuIRE Count output for HEK293T cell transfection were deposited to

517 the NCBI Genome Expression Omnibus with accession number GSE113960. SQuIRE was written in

518 Python2 and is available at the website https://github.com/wyang17/SQuIRE and PyPI. It was developed

519 for UNIX environments. We provide step-by-step instructions on our README to install the correct

520 versions of all software. These instructions include using the package manager Conda (conda.io) to

521 download the correct versions of prerequisite software for SQuIRE (e.g., Python, R (R Development Core

522 Team 2011), STAR, BEDTools, StringTie, SAMtools (Li et al. 2009), UCSC tools and Bioconductor

523 packages. The README also instructs users how to create a non-reference table with the exogenous or

524 polymorphic TE sequences and coordinates that they would like to add to the reference genome. Bash

525 scripts to run each tool in the SQuIRE pipeline are also included. Users can fill in crucial experiment

526 information (raw data, read length, paired, strandedness, genome build, sample name and experimental 
527 design) into the "arguments.sh" file, which the other scripts reference to run each step with the correct

528 parameters.

\section{$529 \quad$ Acknowledgements}

530 We would like to thank Veena Gnanakkan for preparation of C57BL/6 mouse tissue RNA and

531 analysis of Nanostring data. We would like to thank Jane Welch, Paul Schaughency, Shubha Tirumale

532 and Ping Ye for testing SQuIRE. We would like to thank Sibyl Medabalimi for assistance in developing

533 the name of SQuIRE. We would also like to acknowledge the assistance of the NYU Genome Technology

534 Center and Jared Steranka in preparing the RNA for RNA sequencing. This research was supported by

535 National Institutes of Health (NIH) grants R01GM124531 and P50GM107632, and Department of

536 Defense Congressionally Directed Medical Research Program (CDMRP) grant OC120390 (to K.H.B.).

537 W.R.Y. received a Teal Predoctoral Scholar award in association with OC120390.

538 Author Contributions: W.R.Y. contributed to study design, programming of SQuIRE pipeline,

539 statistical analysis, and primary authorship and manuscript; D.A. contributed culture and transfection of

540 HEK293T cells, and suggestions for analysis and manuscript; C.N.P. contributed to debugging SQuIRE

541 and development of README for SQuIRE website; L.M.P. \& K.H.B. jointly contributed to overall study

542 design, data interpretation and manuscript. 


\section{References}

547 Abecasis GR, Auton A, Brooks LD, DePristo M a, Durbin RM, Handsaker RE, Kang HM, Marth GT,

$548 \quad$ McVean G a. 2012. An integrated map of genetic variation from 1,092 human genomes. Nature 491:

$549 \quad 56-65$.

550 http://www.pubmedcentral.nih.gov/articlerender.fcgi?artid=3498066\&tool=pmcentrez\&rendertype= $551 \quad$ abstract (Accessed May 21, 2013).

552 Athanasiadis A, Rich A, Maas S. 2004. Widespread A-to-I RNA editing of Alu-containing mRNAs in the 553 human transcriptome. PLoS Biol 2: e391. http://www.ncbi.nlm.nih.gov/pubmed/15534692

$554 \quad$ (Accessed May 1, 2018).

555 Beck CR, Collier P, Macfarlane C, Malig M, Kidd JM, Eichler EE, Badge RM, Moran J V. 2010. LINE-1

556 retrotransposition activity in human genomes. Cell 141: 1159-70.

557 http://www.ncbi.nlm.nih.gov/pubmed/20602998 (Accessed April 29, 2018).

558 Beck CR, Garcia-Perez JL, Badge RM, Moran J V. 2011. LINE-1 elements in structural variation and 559 disease. Annu Rev Genomics Hum Genet 12: 187-215.

560 http://www.ncbi.nlm.nih.gov/pubmed/21801021 (Accessed April 19, 2018).

561 Boissinot S, Chevret P, Furano A V. 2000. L1 (LINE-1) Retrotransposon Evolution and Amplification in 562 Recent Human History. Mol Biol Evol 17: 915-928. http://academic.oup.com/mbe/article/17/6/915/1037809 (Accessed April 30, 2018).

564 Brawand D, Soumillon M, Necsulea A, Julien P, Csárdi G, Harrigan P, Weier M, Liechti A, Aximu-Petri A, Kircher M, et al. 2011. The evolution of gene expression levels in mammalian organs. Nature 478: 343-348. http://www.nature.com/articles/nature10532 (Accessed April 21, 2018). 
570 Burns KH, Boeke JD. 2012. Human Transposon Tectonics. Cell 149: 740-752. http://linkinghub.elsevier.com/retrieve/pii/S009286741200517X (Accessed August 9, 2017).

Chuong EB, Rumi MAK, Soares MJ, Baker JC. 2013. Endogenous retroviruses function as speciesspecific enhancer elements in the placenta. Nat Genet 45: 325-9. http://www.ncbi.nlm.nih.gov/pubmed/23396136 (Accessed April 21, 2018).

Criscione SW, Zhang Y, Thompson W, Sedivy JM, Neretti N. 2014. Transcriptional landscape of repetitive elements in normal and cancer human cells. BMC Genomics 15: 583.

Deininger P. 2011. Alu elements: know the SINEs. Genome Biol 12: 236. http://genomebiology.biomedcentral.com/articles/10.1186/gb-2011-12-12-236 (Accessed April 19,

Deininger P, Morales ME, White TB, Baddoo M, Hedges DJ, Servant G, Srivastav S, Smither ME, Concha M, DeHaro DL, et al. 2017. A comprehensive approach to expression of L1 loci. Nucleic

Dobin A, Davis CA, Schlesinger F, Drenkow J, Zaleski C, Jha S, Batut P, Chaisson M, Gingeras TR. 2013. STAR: ultrafast universal RNA-seq aligner. Bioinformatics 29: 15-21. https://academic.oup.com/bioinformatics/article-lookup/doi/10.1093/bioinformatics/bts635 
May 1, 2018).

593 Ewing AD, Kazazian HH, Jr. 2010. High-throughput sequencing reveals extensive variation in human-

$594 \quad$ specific L1 content in individual human genomes. Genome Res 20: 1262-70. http://www.ncbi.nlm.nih.gov/pubmed/20488934 (Accessed April 29, 2018).

Ewing AD, Kazazian HH, Jr. 2011. Whole-genome resequencing allows detection of many rare LINE-1 insertion alleles in humans. Genome Res 21: 985-90. http://www.ncbi.nlm.nih.gov/pubmed/20980553 (Accessed April 29, 2018).

Faulkner GJ, Kimura Y, Daub CO, Wani S, Plessy C, Irvine KM, Schroder K, Cloonan N, Steptoe AL, Lassmann T, et al. 2009. The regulated retrotransposon transcriptome of mammalian cells. Nat Consortium 1000 Genomes Project, Devine SE. 2017. The Mobile Element Locator Tool (MELT): population-scale mobile element discovery and biology. Genome Res 27: 1916-1929.

Giordano J, Ge Y, Gelfand Y, Abrusán G, Benson G, Warburton PE. 2007. Evolutionary history of http://www.ncbi.nlm.nih.gov/pubmed/28855259 (Accessed April 27, 2018).

610 Gnanakkan VP, Jaffe AE, Dai L, Fu J, Wheelan SJ, Levitsky HI, Boeke JD, Burns KH. 2013. TE-array--a

611 high throughput tool to study transposon transcription. BMC Genomics 14: 869. http://www.ncbi.nlm.nih.gov/pubmed/24325565 (Accessed April 17, 2018).

613 Hancks DC, Kazazian HH, Jr. 2010. SVA retrotransposons: Evolution and genetic instability. Semin 614 Cancer Biol 20: 234-45. http://www.ncbi.nlm.nih.gov/pubmed/20416380 (Accessed April 19, 
2018).

616 Huang CRL, Burns KH, Boeke JD. 2012. Active Transposition in Genomes. Annu Rev Genet 46: 651-

617 675. http://www.annualreviews.org/doi/10.1146/annurev-genet-110711-155616 (Accessed August

$6189,2017)$

619 Huber W, Carey VJ, Gentleman R, Anders S, Carlson M, Carvalho BS, Bravo HC, Davis S, Gatto L,

620 Girke T, et al. 2015. Orchestrating high-throughput genomic analysis with Bioconductor. Nat

621 Methods 12: 115-21. http://dx.doi.org/10.1038/nmeth.3252 (Accessed February 23, 2016).

622 Iskow RC, McCabe MT, Mills RE, Torene S, Pittard WS, Neuwald AF, Van Meir EG, Vertino PM,

623 Devine SE. 2010. Natural mutagenesis of human genomes by endogenous retrotransposons. Cell

624 141: 1253-61. http://www.ncbi.nlm.nih.gov/pubmed/20603005 (Accessed April 29, 2018).

625 Jin Y, Tam OH, Paniagua E, Hammell M. 2015. TEtranscripts: a package for including transposable elements in differential expression analysis of RNA-seq datasets. Bioinformatics 31: 3593-9.

Kalitsis P, Saffery R. 2009. Inherent promoter bidirectionality facilitates maintenance of sequence http://bioinformatics.oxfordjournals.org/content/early/2015/07/22/bioinformatics.btv422.abstract

Kazazian HH. 2004. Mobile elements: drivers of genome evolution. Science 303: 1626-32. http://science.sciencemag.org/content/303/5664/1626.abstract (Accessed December 22, 2015).

635 Keane TM, Wong K, Adams DJ. 2013. RetroSeq: transposable element discovery from next-generation 636 sequencing data. Bioinformatics 29: 389-90. http://www.ncbi.nlm.nih.gov/pubmed/23233656 $637 \quad$ (Accessed April 27, 2018). 
638 Kent WJ, Sugnet CW, Furey TS, Roskin KM, Pringle TH, Zahler AM, Haussler D. 2002. The human genome browser at UCSC. Genome Res 12: 996-1006.

641 Kimberland ML, Divoky V, Prchal J, Schwahn U, Berger W, Kazazian HH. 1999. Full-Length Human L1

642 Insertions Retain the Capacity for High Frequency Retrotransposition in Cultured Cells. Hum Mol

643 Genet 8: 1557-1560. https://academic.oup.com/hmg/article-lookup/doi/10.1093/hmg/8.8.1557

$644 \quad$ (Accessed April 29, 2018).

645 Lander ES, Linton LM, Birren B, Nusbaum C, Zody MC, Baldwin J, Devon K, Dewar K, Doyle M,

646 FitzHugh W, et al. 2001. Initial sequencing and analysis of the human genome. Nature 409: 860-

647 921. http://dx.doi.org/10.1038/35057062 (Accessed July 10, 2014).

648 Langmead B, Salzberg SL. 2012. Fast gapped-read alignment with Bowtie 2. Nat Methods 9: 357-9.

649 http://www.pubmedcentral.nih.gov/articlerender.fcgi?artid=3322381\&tool=pmcentrez\&rendertype= $650 \quad$ abstract (Accessed July 10, 2014).

651 Langmead B, Trapnell C, Pop M, Salzberg SL. 2009. Ultrafast and memory-efficient alignment of short 652 DNA sequences to the human genome. Genome Biol 10: R25.

653 http://www.pubmedcentral.nih.gov/articlerender.fcgi?artid=2690996\&tool=pmcentrez\&rendertype= 654 abstract (Accessed July 9, 2014).

655 Le TN, Miyazaki Y, Takuno S, Saze H. 2015. Epigenetic regulation of intragenic transposable elements 656 impacts gene transcription in Arabidopsis $\square$ thaliana. Nucleic Acids Res 43: 3911-21.

657 http://nar.oxfordjournals.org/content/early/2015/03/26/nar.gkv258.full (Accessed March 4, 2016).

658 Lee E, Iskow R, Yang L, Gokcumen O, Haseley P, Luquette LJ, Lohr JG, Harris CC, Ding L, Wilson RK, 659 et al. 2012. Landscape of somatic retrotransposition in human cancers. Science 337: 967-71.

660 http://www.ncbi.nlm.nih.gov/pubmed/22745252 (Accessed April 27, 2018). 
661 Lee J, Cordaux R, Han K, Wang J, Hedges DJ, Liang P, Batzer MA. 2007. Different evolutionary fates of recently integrated human and chimpanzee LINE-1 retrotransposons. Gene 390: 18-27.

Lerat E, Fablet M, Modolo L, Lopez-Maestre H, Vieira C. 2016. TEtools facilitates big data expression analysis of transposable elements and reveals an antagonism between their activity and that of piRNA genes. Nucleic Acids Res 45: gkw953. https://academic.oup.com/nar/article-

Li B, Dewey CN. 2011. RSEM: accurate transcript quantification from RNA-Seq data with or without a reference genome. BMC Bioinformatics 12: 323.

Li B, Ruotti V, Stewart RM, Thomson JA, Dewey CN. 2010. RNA-Seq gene expression estimation with http://bmcbioinformatics.biomedcentral.com/articles/10.1186/1471-2105-12-323 (Accessed April read mapping uncertainty. Bioinformatics 26: 493-500.

Love MI, Huber W, Anders S. 2014. Moderated estimation of fold change and dispersion for RNA-seq data with DESeq2. Genome Biol 15: 550. http://www.ncbi.nlm.nih.gov/pubmed/25516281 insertions in humans. Nucleic Acids Res 43: D43-7. http://www.ncbi.nlm.nih.gov/pubmed/25352549 
(Accessed May 1, 2018).

686 Payer LM, Steranka JP, Yang WR, Kryatova M, Medabalimi S, Ardeljan D, Liu C, Boeke JD,

687 Avramopoulos D, Burns KH. 2017. Structural variants caused by Alu insertions are associated with

$688 \quad$ risks for many human diseases. Proc Natl Acad Sci U S A 114: E3984-E3992.

689 http://www.ncbi.nlm.nih.gov/pubmed/28465436 (Accessed April 30, 2018).

690 Perepelitsa-Belancio V, Deininger P. 2003. RNA truncation by premature polyadenylation attenuates

691 human mobile element activity. Nat Genet 35: 363-366. http://www.nature.com/articles/ng1269

$692 \quad$ (Accessed May 1, 2018).

693 Pertea M, Pertea GM, Antonescu CM, Chang T-C, Mendell JT, Salzberg SL. 2015. StringTie enables

694 improved reconstruction of a transcriptome from RNA-seq reads. Nat Biotechnol 33: 290-295.

695 http://www.nature.com/articles/nbt.3122 (Accessed April 20, 2018).

696 Philippe C, Vargas-Landin DB, Doucet AJ, van Essen D, Vera-Otarola J, Kuciak M, Corbin A,

697 Nigumann P, Cristofari G. 2016. Activation of individual L1 retrotransposon instances is restricted

698 to cell-type dependent permissive loci. Elife 5: e13926. https://elifesciences.org/content/5/e13926v1

699 (Accessed March 29, 2016).

700 Pitkänen E, Cajuso T, Katainen R, Kaasinen E, Välimäki N, Palin K, Taipale J, Aaltonen LA, Kilpivaara

701 O. 2014. Frequent L1 retrotranspositions originating from TTC28 in colorectal cancer. Oncotarget

702 5: 853-9. http://www.ncbi.nlm.nih.gov/pubmed/24553397 (Accessed April 29, 2018).

703 Pruitt KD, Brown GR, Hiatt SM, Thibaud-Nissen F, Astashyn A, Ermolaeva O, Farrell CM, Hart J,

704 Landrum MJ, McGarvey KM, et al. 2014. RefSeq: an update on mammalian reference sequences.

$705 \quad$ Nucleic Acids Res 42: D756-63.

706 http://www.pubmedcentral.nih.gov/articlerender.fcgi?artid=3965018\&tool=pmcentrez\&rendertype=

707 abstract (Accessed February 16, 2016). 
Quinlan AR, Hall IM. 2010. BEDTools: a flexible suite of utilities for comparing genomic features. (Accessed July 9, 2014).

711 R Development Core Team R. 2011. R: A Language and Environment for Statistical Computing ed.

$712 \quad$ R.D.C. Team. R Found Stat Comput 1: 409. http://www.r-project.org.

713 Robinson JT, Thorvaldsdóttir H, Winckler W, Guttman M, Lander ES, Getz G, Mesirov JP. 2011.

714 Integrative genomics viewer. Nat Biotechnol 29: 24-6. http://dx.doi.org/10.1038/nbt.1754

$715 \quad$ (Accessed November 19, 2014).

716 Rodić N, Steranka JP, Makohon-Moore A, Moyer A, Shen P, Sharma R, Kohutek ZA, Huang CR, Ahn D,

717 Mita P, et al. 2015. Retrotransposon insertions in the clonal evolution of pancreatic ductal

718 adenocarcinoma. Nat Med 21: 1060-4. http://www.ncbi.nlm.nih.gov/pubmed/26259033 (Accessed

$719 \quad$ April 27, 2018).

720 Rosenbloom KR, Armstrong J, Barber GP, Casper J, Clawson H, Diekhans M, Dreszer TR, Fujita PA,

721 Guruvadoo L, Haeussler M, et al. 2014. The UCSC Genome Browser database: 2015 update.

$722 \quad$ Nucleic Acids Res 43: D670-81.

$723 \mathrm{http} / /$ www.pubmedcentral.nih.gov/articlerender.fcgi?artid=4383971\&tool=pmcentrez\&rendertype=

$724 \quad$ abstract (Accessed January 7, 2015).

725 Saito T, Rehmsmeier M. 2015. The Precision-Recall Plot Is More Informative than the ROC Plot When

726 Evaluating Binary Classifiers on Imbalanced Datasets ed. G. Brock. PLoS One 10: e0118432.

727 http://dx.plos.org/10.1371/journal.pone.0118432 (Accessed April 17, 2018).

728 Schwahn U, Lenzner S, Dong J, Feil S, Hinzmann B, van Duijnhoven G, Kirschner R, Hemberger M,

729 Bergen AAB, Rosenberg T, et al. 1998. Positional cloning of the gene for X-linked retinitis

730 pigmentosa 2. Nat Genet 19: 327-332. http://www.nature.com/articles/ng0898_327 (Accessed April

$73129,2018)$. 
732 Scott EC, Gardner EJ, Masood A, Chuang NT, Vertino PM, Devine SE. 2016. A hot L1 retrotransposon 733 evades somatic repression and initiates human colorectal cancer. Genome Res 26: 745-55.

734 http://www.ncbi.nlm.nih.gov/pubmed/27197217 (Accessed April 19, 2018).

735 Smit, AFA, Hubley, R \& Green P. RepeatMasker Open-4.0. 2013-2015. http://www.repeatmasker.org $736 \quad$ (Accessed April 21, 2018).

737 Smit AFA, Tóth G, Riggs AD, Jurka J. 1995. Ancestral, Mammalian-wide Subfamilies of LINE-1

738 Repetitive Sequences. J Mol Biol 246: 401-417.

739 https://www.sciencedirect.com/science/article/pii/S0022283684700957?via\%3Dihub (Accessed $740 \quad$ April 30, 2018).

741 Sorek R, Ast G, Graur D. 2002. Alu-containing exons are alternatively spliced. Genome Res 12: 1060-7.

742 http://www.ncbi.nlm.nih.gov/pubmed/12097342 (Accessed May 1, 2018).

743 Stewart C, Kural D, Strömberg MP, Walker JA, Konkel MK, Stütz AM, Urban AE, Grubert F, Lam 744 HYK, Lee WP, et al. 2011. A comprehensive map of mobile element insertion polymorphisms in 745 humans ed. H.S. Malik. PLoS Genet 7: e1002236. http://www.ncbi.nlm.nih.gov/pubmed/21876680 $746 \quad$ (Accessed April 19, 2018).

747 Stower H. 2013. Alternative splicing: Regulating Alu element “exonization”. Nat Rev Genet 14: 152-3. 748 http://dx.doi.org/10.1038/nrg3428 (Accessed March 4, 2016).

749 Sudmant PH, Rausch T, Gardner EJ, Handsaker RE, Abyzov A, Huddleston J, Zhang Y, Ye K, Jun G, $750 \quad$ Hsi-Yang Fritz M, et al. 2015. An integrated map of structural variation in 2,504 human genomes. $751 \quad$ Nature 526: 75-81. http://www.nature.com/doifinder/10.1038/nature15394 (Accessed April 19, 752 2018).

753 Taylor MS, LaCava J, Mita P, Molloy KR, Huang CRL, Li D, Adney EM, Jiang H, Burns KH, Chait BT, 754 et al. 2013. Affinity Proteomics Reveals Human Host Factors Implicated in Discrete Stages of 

(Accessed April 20, 2018).

757

Tubio JMC, Li Y, Ju YS, Martincorena I, Cooke SL, Tojo M, Gundem G, Pipinikas CP, Zamora J, Raine K, et al. 2014. Mobile DNA in cancer. Extensive transduction of nonrepetitive DNA mediated by L1 retrotransposition in cancer genomes. Science 345: 1251343. http://www.ncbi.nlm.nih.gov/pubmed/25082706 (Accessed April 29, 2018).

Upton KR, Gerhardt DJ, Jesuadian JS, Richardson SR, Sánchez-Luque FJ, Bodea GO, Ewing AD, Salvador-Palomeque C, van der Knaap MS, Brennan PM, et al. 2015. Ubiquitous L1 mosaicism in hippocampal neurons. Cell 161: 228-39. http://www.ncbi.nlm.nih.gov/pubmed/25860606 (Accessed April 27, 2018).

Wang J, Song L, Grover D, Azrak S, Batzer MA, Liang P. 2006. dbRIP: A highly integrated database of retrotransposon insertion polymorphisms in humans. Hum Mutat 27: 323-329. http://www.ncbi.nlm.nih.gov/pubmed/16511833 (Accessed May 1, 2018).

Wicker T, Sabot F, Hua-Van A, Bennetzen JL, Capy P, Chalhoub B, Flavell A, Leroy P, Morgante M, Panaud O, et al. 2007. A unified classification system for eukaryotic transposable elements. Nat Rev Genet 8: 973-82. http://dx.doi.org/10.1038/nrg2165 (Accessed March 15, 2016).

Xie M, Hong C, Zhang B, Lowdon RF, Xing X, Li D, Zhou X, Lee HJ, Maire CL, Ligon KL, et al. 2013. DNA hypomethylation within specific transposable element families associates with tissue-specific enhancer landscape. Nat Genet 45: 836-41. http://dx.doi.org/10.1038/ng.2649 (Accessed July 22, 2015).

Yue F, Cheng Y, Breschi A, Vierstra J, Wu W, Ryba T, Sandstrom R, Ma Z, Davis C, Pope BD, et al. 2014. A comparative encyclopedia of DNA elements in the mouse genome. Nature 515: 355-364. http://www.ncbi.nlm.nih.gov/pubmed/25409824 (Accessed April 18, 2018). 
bioRxiv preprint doi: https://doi.org/10.1101/313999; this version posted May 4 2018. The copyright holder for this preprint (which was not certified by peer review) is the author/funder, who has granted bioRxiv a license to display the preprint in perpetuity. It is made available under aCC-BY-NC 4.0 International license.

778

779

780 